\title{
Perfil de prematuros em atendimento fonoaudiológico em um ambulatório de follow up
}

\section{Profile of premature infants undergoing speech-language-hearing}

\author{
care at a follow-up outpatient center
}

\author{
Maisa Alves Teixeira' (D), Denise Brandão de Oliveira e Britto² (D), Andrezza Gonzalez Escarce ${ }^{2}$ (D), \\ Danielle Diniz de Paula ${ }^{1}$, Stela Maris Aguiar Lemos² ${ }^{2}$
}

\section{RESUMO}

Objetivo: descrever o perfil de crianças pré-termo, segundo aspectos sociodemográficos, clínicos e assistenciais, e a associação com dados perinatais e pós-natais. Métodos: estudo observacional descritivo transversal, aprovado pelo Comitê de Ética em Pesquisa da Universidade Federal de Minas Gerais - CEP-UFMG, sob o Parecer 3.615.440, realizado por meio da análise de 749 prontuários de crianças pré-termo, atendidas no setor de Fonoaudiologia de ambulatório multidisciplinar de follow-up. Foram incluídos no estudo todos os prontuários do período de 2009 a 2019. Para as análises de associação foram utilizados os testes Qui-quadrado de Pearson e Kruskal-Wallis, sendo considerados como valores com significância estatística os que apresentaram valor de $p \leq 0,05$. Resultados: verificou-se distribuição similar entre os sexos feminino e masculino e a maior parte das crianças com prematuridade moderada a tardia. A maioria dos pais apresentou ensino médio completo, com renda familiar de até dois salários mínimos $(60,0 \%)$. Quanto aos aspectos clínicos, observou-se significância estatística $(\mathrm{p} \leq 0,001)$ na associação da idade gestacional com as seguintes variáveis: peso ao nascimento, estatura, perímetro cefálico, uso do Método Canguru, alimentação na alta, drogas ototóxicas, hemorragia intracraniana, sepse e icterícia. A maioria dos bebês $(99,9 \%)$ realizou o Teste Guthrie com resultado adequado $(95,3 \%)$. Da mesma forma, referente à realização da Triagem Auditiva Neonatal - (79,9\%), com resultado adequado $(89,4 \%)$. Conclusão: a maioria das famílias era de baixa renda, com bebês apresentando prematuridade tardia. Observou-se significância estatística para as variáveis: tipo de parto, peso, estatura, perímetro cefálico, uso de drogas ototóxicas, hemorragia intracraniana, sepse e icterícia, com alterações predominantes em prematuros extremos.

Palavras-chave: Fonoaudiologia; Perfil de Saúde; Fatores de Risco; Recém-Nascido Prematuro; Saúde da criança; Assistência Ambulatorial

\begin{abstract}
Purpose: to describe the profile of preterm children based on sociodemographic, clinical, and assistance aspects, and their association with peri- and postnatal data. Methods: observational, descriptive, cross-sectional study, approved by the Research Ethics Committee of the Universidade Federal de Minas Gerais (UFMG, Brazil), under evaluation report no. 3,615.440. It was based on the analysis of 749 medical records of preterm children attended at the speech-language-hearing department of a multidisciplinary follow-up outpatient center. All medical records from 2009 to 2019 were included in the study. The association analyses were made with the Pearson chi-square and Kruskal-Wallis tests, and the statistically significant values were set at $\mathrm{p} \leq 0.05$. Results: the sample was evenly distributed between females and males, and most of them were moderate-to-late premature children. Most parents had graduated from high school, earning up to two minimum wages $(60.0 \%)$. As for the clinical aspects, there was a statistically significant association $(\mathrm{p} \leq 0.001)$ between gestational age and birth weight, length, head circumference, use of kangaroo care, feeding method at discharge, ototoxic drugs, intracranial hemorrhage, sepsis, and jaundice. Most babies (99.9\%) were submitted to the Guthrie test, with normal results $(95.3 \%)$. It was likewise with the Neonatal Hearing Screening (79.9\%), with normal results (89.4\%). Conclusion: most of the sample were from low-income families, with late premature babies. There was a statistical significance for type of delivery, weight, length, head circumference, ototoxic drug use, intracranial hemorrhage, sepsis, and jaundice, with changes occurring predominantly in extremely premature infants.
\end{abstract}

Keywords: Speech Therapy; Health Profile; Risk Factors; Premature Newborn; Child Health; Ambulatory Care

\footnotetext{
Trabalho realizado no Programa de Pós-graduação em Ciências Fonoaudiológicas, Universidade Federal de Minas Gerais - UFMG - Belo Horizonte (MG), Brasil.

${ }^{1}$ Programa de Pós-graduação em Ciências Fonoaudiológicas, Universidade Federal de Minas Gerais - UFMG - Belo Horizonte (MG), Brasil.

${ }^{2}$ Departamento de Fonoaudiologia, Universidade Federal de Minas Gerais - UFMG - Belo Horizonte (MG), Brasil.

Conflito de interesses: Não.

Contribuição dos autores: MAT participou da concepção do estudo, coleta e análise de dados, redação do manuscrito e aprovação da versão final; DBOB e AGE participaram, na condição de coorientadoras, da concepção do estudo, análise e interpretação dos dados, redação do manuscrito e aprovação da versão final; DDP participou da coleta e análise dos dados e aprovação da versão final; SMAL participou, na condição de orientadora, da concepção do estudo, análise e interpretação dos dados, redação do manuscrito e aprovação da versão final.

Financiamento: Coordenação de Aperfeiçoamento de Pessoal de Nível Superior - Brasil (CAPES) - Código de Financiamento 001.

Autor correspondente: Stela Maris Aguiar Lemos. E-mail: lemos.stela@gmail.com
}

Recebido: Novembro 13, 2020; Aceito: Novembro 11, 2021 


\section{INTRODUÇÃO}

Tendo em vista os riscos à saúde, a população oriunda do nascimento prematuro tem sido estudada, devido à chance de comprometimento no desenvolvimento e à necessidade de cuidados especializados ${ }^{(1)}$.

São considerados prematuros ou pré-termo os bebês que nascem com idade gestacional menor ou igual a 37 semanas. De acordo com a Organização Mundial de Saúde (OMS) ${ }^{(2)}$, a prematuridade é classificada em moderada à tardia (nascidos entre 32 e 37 semanas de idade gestacional), muito prematuros (nascidos entre 28 e 32 semanas) e prematuros extremos (nascidos com menos de 27 semanas de idade gestacional). Atualmente, são registrados no mundo, cerca de 15 milhões de nascimentos prematuros por ano e o Brasil está entre os dez países com maior número de $\operatorname{casos}^{(2)}$.

Os bebês prematuros são mais suscetíveis aos cuidados intensivos nos períodos perinatal e pós-natal, visto que intercorrências nesses períodos poderão acarretar prejuízos futuros no seu desenvolvimento ${ }^{(3)}$. Assim, torna-se necessária a melhor assistência ao pré-termo, tanto na terapia intensiva, quanto no cuidado continuado.

A prestação de serviço à saúde requer conhecimento do perfil epidemiológico e assistencial da população atendida e, portanto, o atendimento será aprimorado e o profissional da saúde irá adaptar seus serviços às demandas dos pacientes.

A pesquisa sobre o perfil do paciente assistido possibilitará conhecimento de fatores pertinentes ao nascimento prematuro, tais como alteração do índice de APGAR, baixo peso ao nascimento, administração de ototóxicos, internação em Unidade de Tratamento Intensivo (UTI), dentre outros, que podem acarretar mudanças motoras e cognitivas ${ }^{(3-5)}$. Sendo assim, a criança prematura poderá precisar de intervenção multidisciplinar para garantia de seu desenvolvimento.

Diante disso, conhecer os fatores associados ao nascimento prematuro é necessário para a compreensão dos prováveis riscos para distúrbios do desenvolvimento, garantindo, assim, a intervenção especializada e humanizada.

O presente estudo teve como objetivo descrever o perfil de crianças com histórico de prematuridade, atendidas pela equipe de Fonoaudiologia em um ambulatório de follow-up, segundo aspectos sociodemográficos, clínicos e assistenciais, e a associação com dados perinatais e pós-natais.

\section{MÉTODOS}

Trata-se de estudo observacional descritivo de caráter transversal, realizado por meio da análise de prontuários de crianças com histórico de prematuridade. O projeto foi aprovado pelo Comitê de Ética em Pesquisa da Universidade Federal de Minas Gerais - CEP-UFMG, sob parecer n ${ }^{\circ} 3.615 .440$. Por se tratar de pesquisa com dados secundários, houve a dispensa do Termo de Consentimento Livre e Esclarecido. Os prontuários analisados procediam do setor de Fonoaudiologia de um ambulatório de follow-up, situado em um hospital universitário. O ambulatório realiza o acompanhamento de recém-nascidos pré-termo, desde o nascimento até os 7 anos de idade e conta com equipe constituída por pediatras, neuropediatras, enfermeiras, terapeutas ocupacionais, fisioterapeutas, fonoaudiólogos e psicólogos $^{(6)}$.
Foram incluídos todos os prontuários do período de maio de 2009 a novembro de 2019 e os dados coletados referiamse, especificamente, à primeira consulta da criança no serviço de Fonoaudiologia. Deste modo, a faixa etária da amostra compreendeu prematuros de 1 a 24 meses e foram analisados 749 prontuários. No entanto, houve alteração desse número em algumas variáveis, devido à ausência de informações nos prontuários.

Para análise dos dados, foram consultadas informações da anamnese aplicada aos pais no ingresso ao serviço de Fonoaudiologia, contemplando três eixos: a) dados sociodemográficos: perfil de moradia, renda familiar, presença de saneamento básico e eletricidade, idade, profissão e instrução parental, número de irmãos; b) aspectos clínicos: informações do período gestacional, informações e intercorrências referentes aos períodos pré-natal, perinatal e pós-natal; c) aspectos assistenciais: acesso à saúde e realização de triagens neonatais.

As informações contidas nos prontuários foram digitadas em banco de dados organizado no Excel®. Foi realizada análise descritiva dos dados, com distribuição de frequência das variáveis categóricas e análise das medidas de tendência central e de dispersão das variáveis contínuas. Para as análises de associação, foram utilizados os testes Qui-quadrado de Pearson e Kruskal-Wallis, sendo considerados como valores com significância estatística os que apresentaram $\mathrm{p} \leq 0,05$.

Para realizar os cálculos estatísticos foi utilizado o programa SPSS (Statistical Package for the Social Sciences), versão 21.0

\section{RESULTADOS}

Os resultados referem-se aos dados registrados na primeira consulta no serviço de Fonoaudiologia, na faixa etária de 1 a 24 meses, com média de 2 meses para idade corrigida e 6 meses para idade cronológica. A análise descritiva mostrou distribuição similar entre os sexos feminino e masculino (49,8\% e 50,2\%, respectivamente) e que a maior parte das crianças nasceu com idade gestacional entre 32 e 37 semanas $(45,8 \%)$, cuja classificação da prematuridade é de moderada a tardia. Quanto ao índice de APGAR a maioria apresentou normalidade ${ }^{(7)}$ (maior que 6), tanto no primeiro $(68,9 \%)$ quanto no quinto minuto $(95,1 \%)$.

Quanto à escolaridade parental, 5,4\% dos pais tinham ensino fundamental I incompleto, $13 \%$, ensino fundamental II incompleto, 48,3\%, ensino médio completo ou superior incompleto e 5,3\% tinham superior completo, enquanto 3,4\% das mães possuíam ensino fundamental I incompleto, 11,4\%, ensino fundamental II incompleto, 54,6\% ensino médio completo ou superior incompleto e 9,5\% tinham ensino superior completo. Quanto à renda, 60,1\% das famílias recebiam até 2 salários mínimos e 13,4\% recebiam acima de 4 salários mínimos. Quanto às condições de moradia, 69\% declararam viver em casa própria, $96,6 \%$ com saneamento básico e $99,9 \%$ contavam com eletricidade.

Vale destacar que a maioria dos pais tinha idade de, aproximadamente, 30 anos, e as mães, 29 anos. Referente ao número de irmãos, observou-se média de um irmão para os bebês prematuros. Não houve significância estatística nas variáveis referentes à idade dos pais e número de irmãos, em associação com a idade gestacional.

A associação entre a idade gestacional e as variáveis referentes ao período gestacional revelou que, dentre os itens analisados, apenas a variável realização de pré-natal apresentou 
significância estatística $(\mathrm{p}=0,044)$. Os dados indicaram que a maior parte foi de bebês nascidos entre 32 e 37 semanas, cujas mães realizaram pré-natal (Tabela 1).

Quanto aos dados do nascimento, verificou-se significância estatística na associação da idade gestacional com as variáveis peso ao nascimento (em gramas), estatura (em centímetros) e perímetro cefálico (em centímetros), que revelaram menor mediana na idade gestacional de 27 semanas ou menos (Figura 1).

Quanto à análise de associação entre a idade gestacional e as variáveis referentes ao período pós-natal, houve significância estatística entre as variáveis: tipo de parto, com maior ocorrência de parto cesáreo em prematuros de 32 a 37 semanas $(p \leq 0,001)$; uso do Método Canguru ( $\mathrm{p} \leq 0,014)$; alimentação na alta, com maior ocorrência de aleitamento materno exclusivo ( $\mathrm{p} \leq 0,001)$; drogas ototóxicas $(p \leq 0,001)$; hemorragia intracraniana $(p \leq 0,001)$; sepse $(p \leq 0,001)$ e icterícia $(p \leq 0,027)$ (Tabela 2$)$.

Referente aos dias de permanência em UTI e no Método Canguru, observou-se significância estatística na associação com a idade gestacional, mostrando maior mediana na idade gestacional de 27 semanas ou menos (Figura 2).

Em relação aos testes realizados no período pós-natal, a maioria dos bebês $(99,9 \%)$ realizou o Teste Guthrie (teste do pezinho) e, desses, a maioria $(95,3 \%)$ apresentou resultado dentro dos padrões de normalidade. Quanto à avaliação do frênulo da língua (teste da linguinha), $82,8 \%$ das famílias não foram questionadas sobre sua realização, compondo a variável "não

Tabela 1. Associação entre as variáveis referentes à idade gestacional e variáveis explicativas

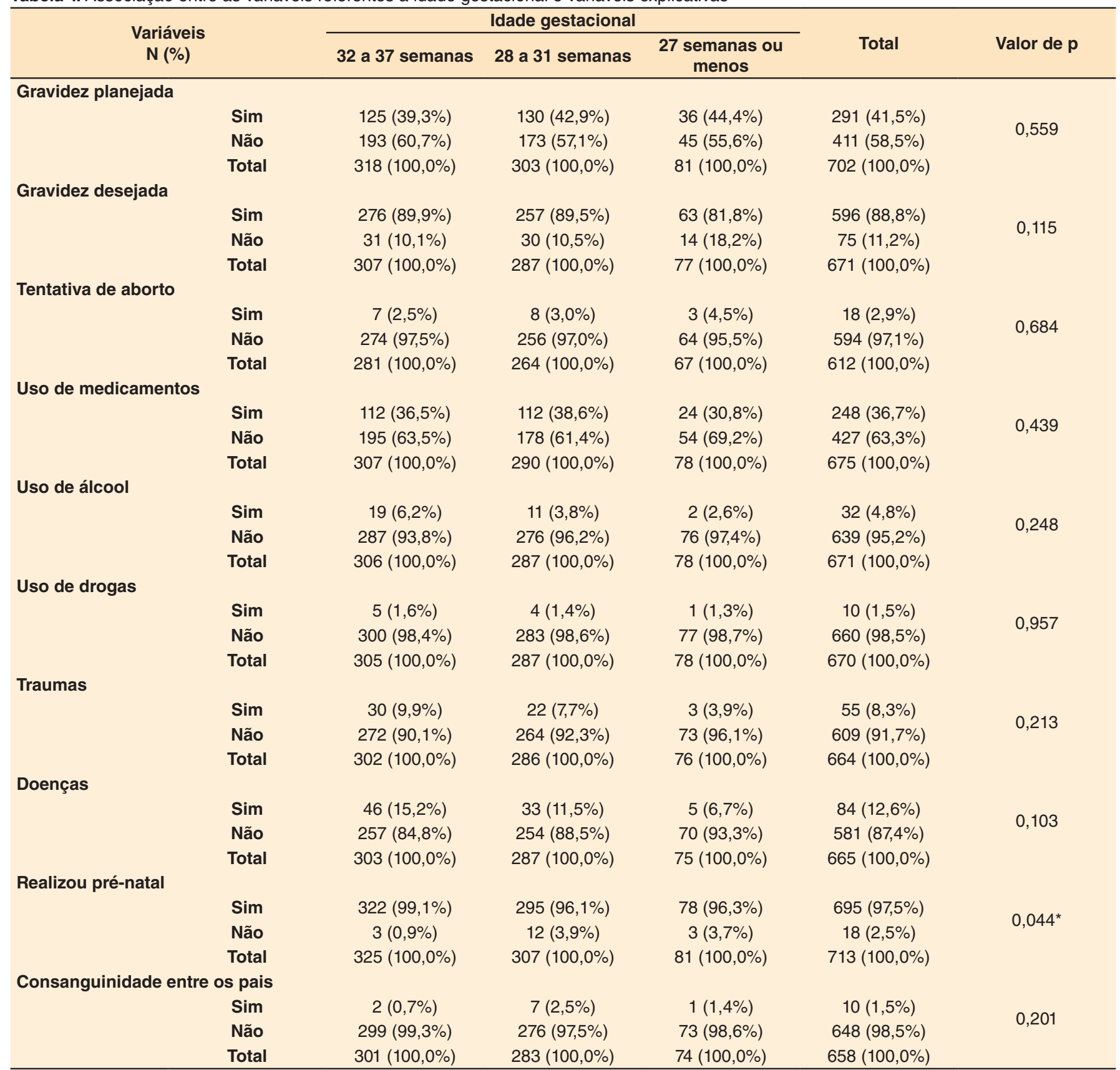

*Valor de $p \leq 0,05$. Teste Qui-quadrado de Pearson

Legenda: $\mathrm{N}$ = número variado, devido à ausência de informações no prontuário; \% = percentual 

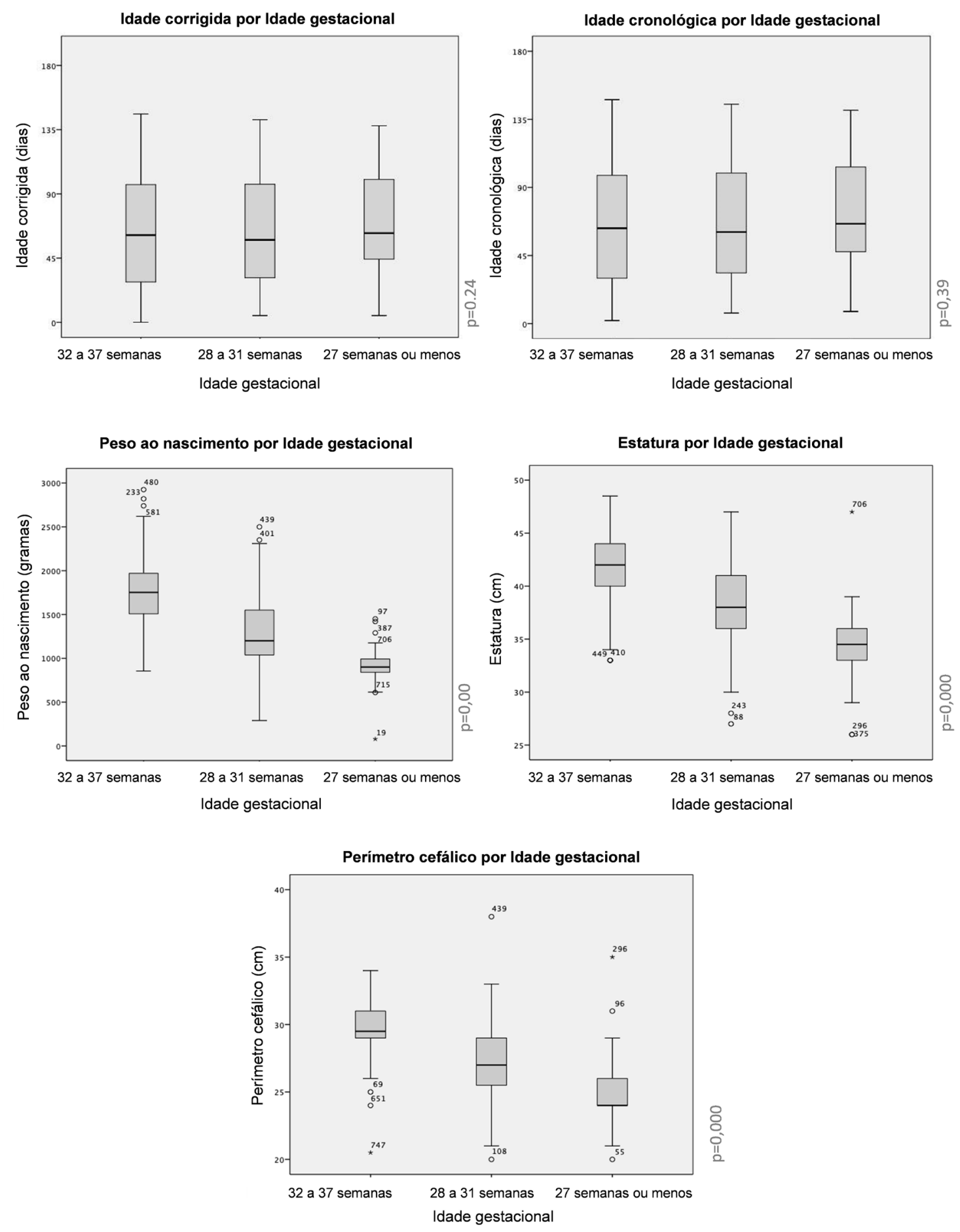

Figura 1. Boxplot entre as variáveis idade gestacional (semanas) e idade corrigida e cronológica (dias), peso ao nascimento (gramas), estatura (centímetros) e perímetro cefálico (centímetros)

Legenda: ${ }_{0}^{*}$ : outliers $=$ valores discrepantes

se aplica". Das famílias que foram questionadas, a maior parte $(13,5 \%)$ relatou não ter realizado o teste. Referente à Triagem Auditiva Neonatal Específica (TANE), 79,8\% realizaram o teste, dos quais, a maioria apresentou resultado "passa" (89,2\%). A análise de associação não mostrou significância estatística entre as variáveis analisadas (Tabela 3).

\section{DISCUSSÃO}

A caracterização do perfil de 749 crianças prematuras, atendidas no setor de Fonoaudiologia do ambulatório de follow-up, no período de dez anos, permitiu a compilação de 
Tabela 2. Associação entre variáveis referentes à idade gestacional e períodos perinatal e pós natal

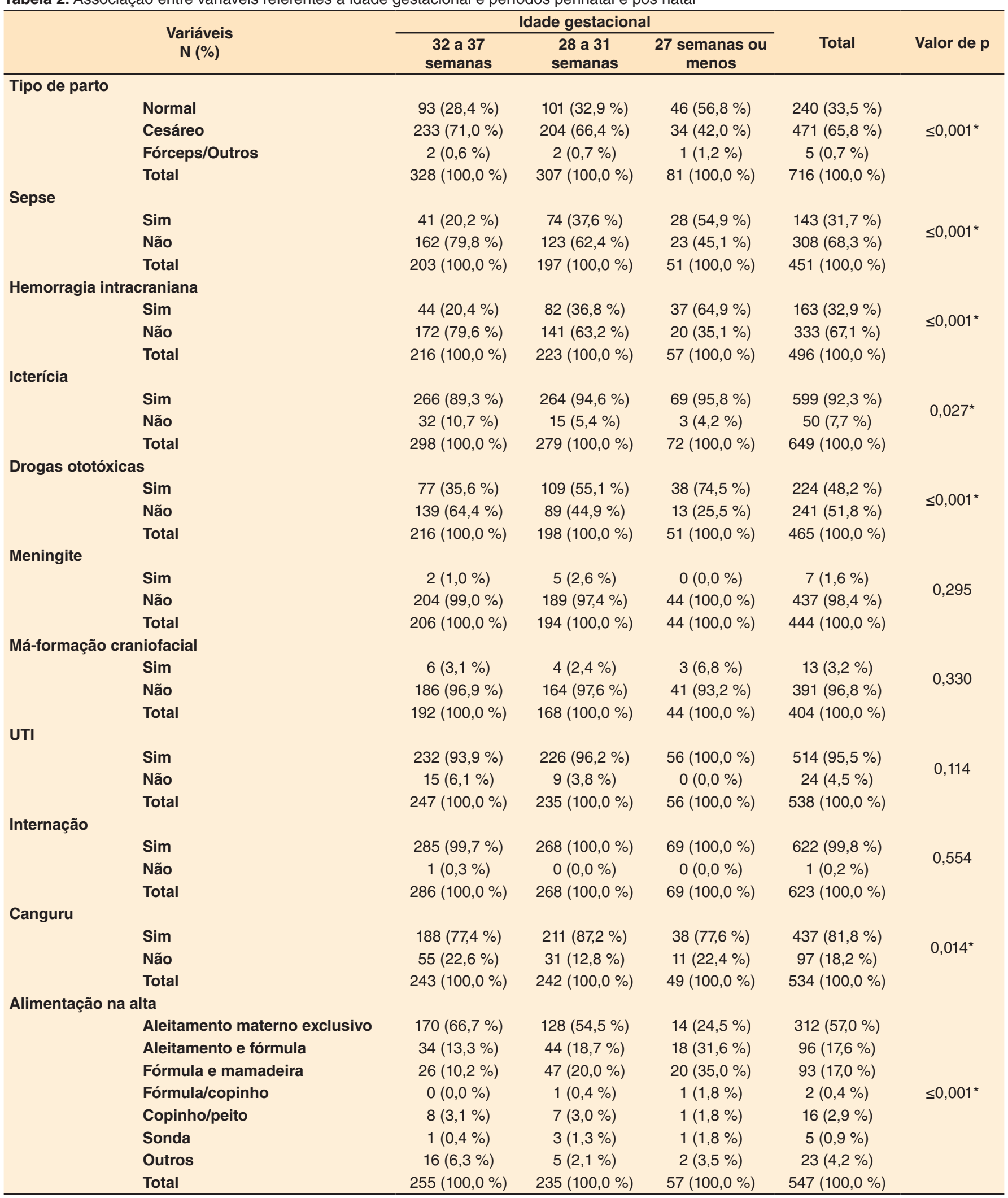

* Valor de $\mathrm{p} \leq$ 0,05. Teste Qui-Quadrado de Pearson

Legenda: $\mathrm{N}$ = número variado, devido à ausência de informações no prontuário; UTI = Unidade de Tratamento Intensivo; \% - percentual 


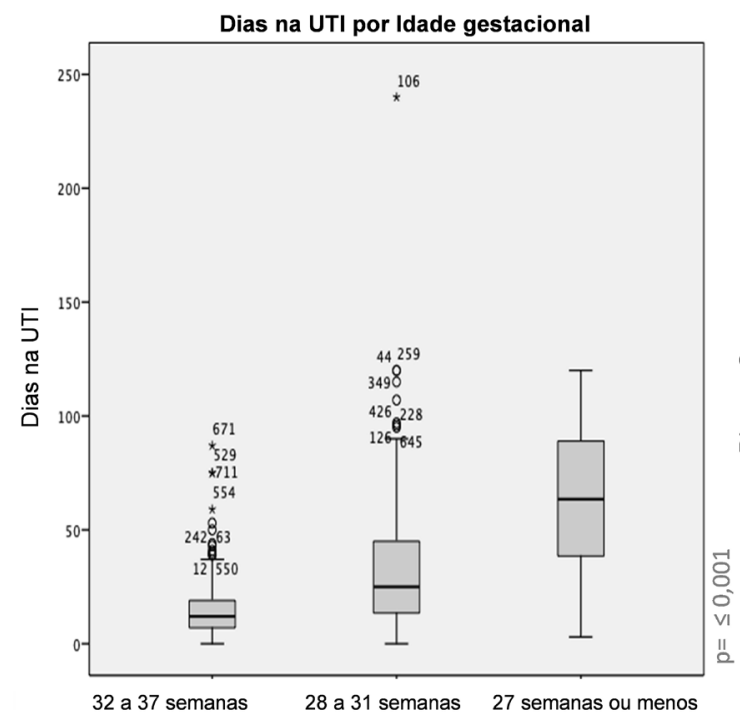

Idade gestacional

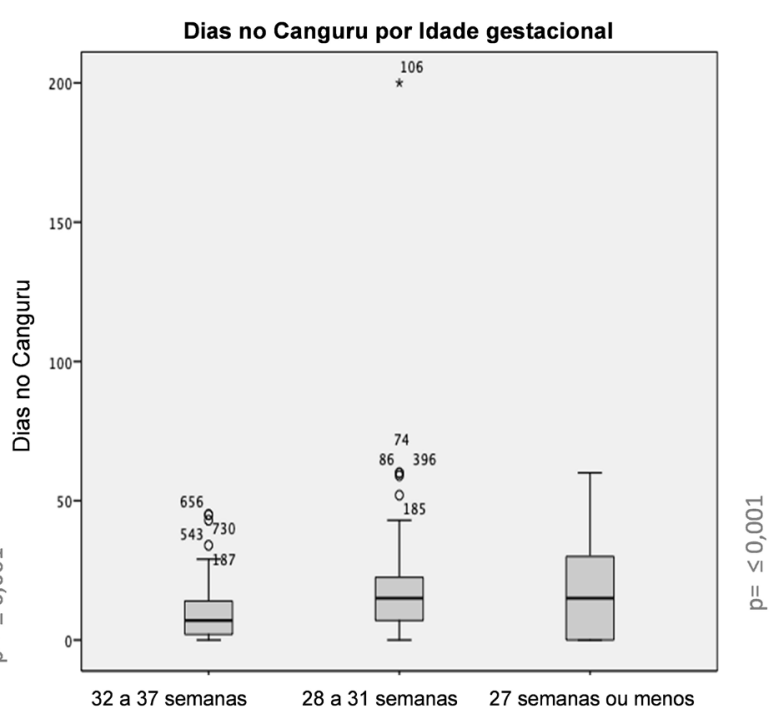

Idade gestacional

Figura 2. Boxplot entre as variáveis idade gestacional e número de permanência na Unidade de Tratamento Intensivo e Método Canguru Legenda: ${ }^{*}$ : outliers $=$ valores discrepantes

Tabela 3. Associação entre testes realizados no período pós-natal e idade gestacional

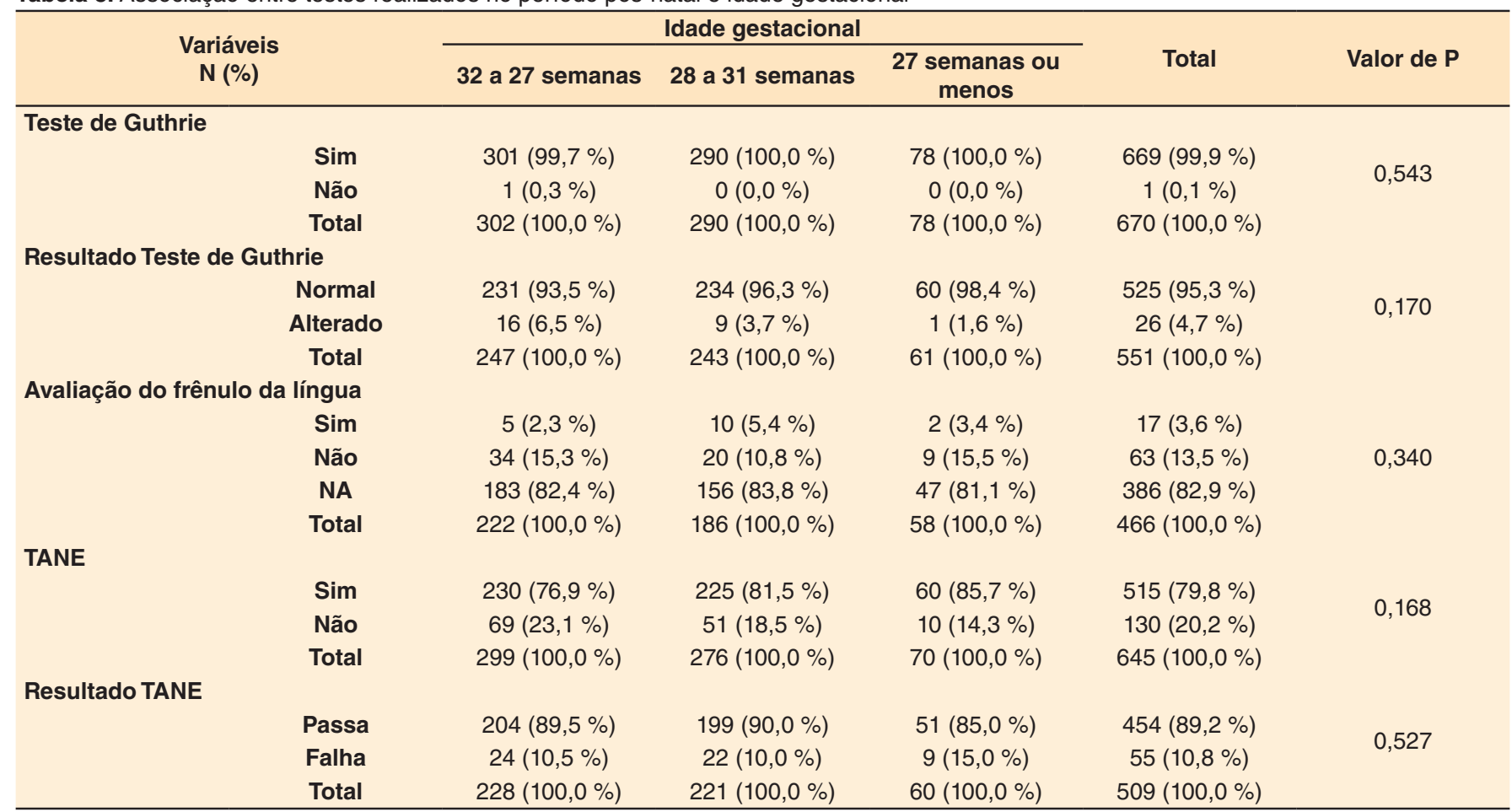

Teste Qui-quadrado de Pearson

Legenda: N = número variado, devido à ausência de informações no prontuário; \% = percentual; NA = não se aplica; TANE = Triagem Auditiva Neonatal Específica

dados sociodemográficos, clínicos e assistenciais da população atendida. O primeiro dado a ser destacado foi a distribuição similar quanto ao sexo, resultado também presente em outros estudos nacionais ${ }^{(1,8)}$.

A caracterização da história clínica indicou que a maioria dos pacientes atendidos, com idade gestacional entre 28 e 37 semanas, apresentou APGAR normal no primeiro minuto. Estudo(4) que mostrou indicadores de risco de prematuros para distúrbios da linguagem apontou associação entre APGAR alterado $^{(7)}$ no primeiro minuto com futuros distúrbios da linguagem. $\mathrm{O}$ dado revela a importância da investigação do APGAR em casos de prematuros, que são mais propensos a tais distúrbios.

Em relação aos aspectos sociodemográficos, verificou-se que mais da metade das mães e dos pais apresentava ensino 
médio completo. Tais resultados são similares aos de outros estudos $^{(9,10)}$, que investigaram a relação da escolaridade parental com a prematuridade. No entanto, não encontraram associações com significância estatística, assim como observado no presente estudo. Contudo, cabe ressaltar o baixo registro da escolaridade materna e paterna na presente pesquisa, pois, dentre os prontuários analisados, menos da metade apresentava esse dado. Estudos ${ }^{(11,12)}$ demonstram a importância da escolaridade parental para o desenvolvimento infantil, assim como a renda familiar, portanto, tais dados são fundamentais para análise e devem estar devidamente registrados nos prontuários.

No presente estudo, a maioria das famílias possuía renda de até dois salários mínimos e residia em casa própria, com saneamento e eletricidade. Estudo prévio ${ }^{(11)}$ revelou que a renda familiar foi inversamente relacionada ao afeto negativo das crianças, mostrando que aspectos como renda e escolaridade parental são indicativos de reações comportamentais negativas das crianças. Vale destacar que o desenvolvimento da criança está ligado, também, aos aspectos ambientais e condições de vida em geral, como evidenciado em revisão de literatura, na qual se concluiu que moradia, saneamento do meio e acesso a serviços básicos de saúde são fatores que contribuem para o desenvolvimento infantil ${ }^{(13)}$.

Neste estudo, foi verificada significância estatística entre a idade gestacional e os itens realização de pré-natal, peso ao nascimento, estatura e perímetro cefálico. Referente ao prénatal, observou-se que, dentre os prontuários com o registro de pré-natal realizado, a maioria era de bebês com idade gestacional de 32 a 37 semanas. Estudos ${ }^{(9,14)}$ realizados no Pará e Rio Grande do Sul mostraram que a ausência de pré-natal, ou número inadequado de sessões, aumentam as chances de parto prematuro. Visto isso, é importante a investigação de tal dado em casos de crianças prematuras, para viabilizar futuras correlações e fatores de risco para o desenvolvimento.

Em relação ao peso ao nascimento, estatura e perímetro cefálico, os resultados revelaram que bebês com idade gestacional de 27 semanas ou menos, classificados como prematuros extremos, apresentaram menor mediana para os itens avaliados. Estudos realizados na Arábia Saudita ${ }^{(15)}$ e Ruanda ${ }^{(3)}$ apontaram dados similares, nos quais houve prevalência de nascidos com baixo peso $(<1000 \mathrm{~g})$ entre bebês com menos de 28 semanas de idade gestacional. Outro estudo nacional ${ }^{(16)}$ citou resultados equivalentes, mostrando baixo peso e baixa estatura em prematuros com menos de 27 semanas.

Em relação ao tipo de parto, o presente estudo demonstrou que a maioria dos bebês nasceu por parto cesáreo, sendo a idade gestacional predominante de 32 a 37 semanas, mostrando significância estatística em tal dado. $\mathrm{O}$ resultado foi similar aos de outros estudos ${ }^{(9,10,16,17)}$, que mostram maior prevalência de parto cesáreo em casos de prematuridade. Desse modo, foi possível constatar que o ambulatório estudado apresenta perfil semelhante aos de outros cenários presentes na literatura.

Referente aos dados sobre intercorrências pós-parto, houve significância estatística nas variáveis sepse, hemorragia intracraniana e icterícia ao nascimento. Alguns estudos ${ }^{(18,19)}$ demonstraram índices elevados de sepse e hemorragia intracraniana em prematuros extremos, mostrando ser fator de risco eminente para mortalidade. Neste estudo, foi possível observar que a maioria das mães que relatou ausência dessas complicações, era de mães de bebês nascidos com prematuridade tardia.

Tendo em vista a ocorrência de maiores complicações em prematuros extremos, os cuidados intensivos, como, por exemplo, o uso de drogas ototóxicas, são mais prevalentes, assim como observado em estudo ${ }^{(5)}$ que mostrou a necessidade do uso de ototóxicos nos cuidados com prematuros extremos. O presente estudo apresentou resultados que concordam com a literatura, mostrando que, dentre os bebês nascidos com idade gestacional de 27 semanas ou menos, a maioria necessitou fazer uso de drogas ototóxicas, revelando significância estatística em tal dado.

As variáveis dias na UTI, e dias no Método Canguru também apresentaram maior mediana para a idade gestacional de 27 semanas ou menos e revelaram significância estatística. Já a variável uso do Método Canguru, apresentou maior mediana para nascidos entre 28 e 31 semanas. Estudo realizado em Brasília ${ }^{(20)}$ mostrou que todos os bebês nascidos com prematuridade extrema necessitaram de internação em Unidade de Tratamento Intensivo Neonatal (UTIN), demostrando que prematuros extremos requerem maior cuidado intensivo. Esses dados concordam com os achados do presente estudo, em que foram encontrados maiores índices de cuidados intensivos para prematuros extremos. Dentre esses cuidados intensivos, ressalta-se a importância do Método Canguru, que favorece mudanças fisiológicas benéficas ao prematuro extremo, diminuindo o tempo de internação ${ }^{(21)}$, o que pode explicar o maior número de dias nesse método em bebês com prematuridade extrema.

A alimentação na alta hospitalar predominante na população estudada foi aleitamento materno exclusivo, sobretudo em bebês nascidos entre 32 e 37 semanas, seguido de aleitamento materno mais fórmula, em bebês nascidos com 27 semanas ou menos. Um estudo paulista ${ }^{(22)}$ revelou resultados semelhantes, no qual a maioria dos recém-nascidos teve alta com aleitamento materno e, destes, $48,3 \%$ com aleitamento materno exclusivo e $38,2 \%$ com aleitamento materno acrescido de fórmula.

A diferença da amamentação natural exclusiva para a amamentação natural acrescida de fórmula, para o ganho de peso do prematuro extremo, é relatada em estudo que constatou que, nos casos de prematuridade extrema, o bebê necessita de maior cuidado, incluindo acréscimo de nutrientes e vitaminas, que pode ser sanado por meio de fórmulas ${ }^{(23)}$. Considerando que a alimentação do bebê prematuro sofrerá influências, devido ao trato intestinal imaturo e/ou dificuldades quanto à coordenação na amamentação ${ }^{(24)}$, o prematuro tardio terá mais facilidade em se adaptar à amamentação exclusiva, comparado ao prematuro extremo, que requer maior necessidade de interferências e complementos em relação à alimentação.

Quanto aos testes de triagem realizados no período pós-natal, é válido ressaltar sua importância no prognóstico e cuidados, visando melhor desenvolvimento da criança. Referente ao Teste de Guthrie (teste do pezinho), destaca-se a relevância para o diagnóstico precoce de doenças congênitas e a viabilidade de início antecipado do tratamento, em casos necessários ${ }^{(25)}$. O teste para avaliação do frênulo da língua (teste da linguinha) se faz necessário no período pós-natal para garantia de desenvolvimento alimentar do bebê, uma vez que alterações do frênulo lingual estão ligadas ao desempenho de sucção e, posteriormente, podem influenciar nos aspectos de fala ${ }^{(26)}$. A Triagem Auditiva Neonatal Específica (TANE) colabora para a detecção precoce de perdas auditivas, preconizando a intervenção em tempo oportuno para garantia do desenvolvimento auditivo e de linguagem da criança ${ }^{(27)}$.

Neste estudo, foi possível observar maior porcentagem de realização do Teste de Guthrie (99,9\%). Estudo ${ }^{25}$ realizado em São Paulo mostrou resultados semelhantes, com registro de 96,3\% de triagens no ano de 2017. Embora existam políticas 
de obrigatoriedade para realização da TANE e do teste da linguinha ${ }^{(28,29)}$, observa-se que ainda existem lacunas para a sua realização de forma abrangente. A falta de compreensão sobre a importância desses testes pode ser fator agravante, diminuindo a prevalência de realização.

Baixo índice de realização da avaliação do frênulo lingual $(3,6 \%)$ foi observado no presente estudo. Destaca-se que a ausência da pergunta em protocolo de anamnese pode ter colaborado com o índice, aumentando o número na variável "não se aplica". Tendo em vista que o teste foi implantado no ano de $2014^{(29)}$, os prontuários estudados que precediam essa data não possuíam tal informação. Além disso, nos casos em que houve relato, os informantes não sabiam indicar tal dado no momento da anamnese, acredita-se que por se tratar de uma implantação não conhecida de forma ampla, como as demais triagens.

Constituiu-se como principal limitação do estudo, o fato de a pesquisa ter sido realizada com prematuros de apenas um ambulatório de referência, limitando, assim, que comparações sociodemográficas e de infraestrutura sejam realizadas. Além disso, destaca-se como limitação a ausência de informações em prontuários, como apontam estudos com metodologia similar $^{(1,3)}$. Acredita-se que a ausência de informações pode ser resultante do viés de memória do informante, do preenchimento pelo profissional, ou da variação de nomenclatura e termos clínicos adotados pelos diferentes profissionais envolvidos no atendimento dos dez anos de análise.

Considera-se que o estudo apresentou avanços, ao estabelecer o perfil da população atendida no setor de Fonoaudiologia de um ambulatório de seguimento (follow-up) em um hospital de ensino de referência. Propiciou conhecimento do perfil sociodemográfico da população assistida para, assim, otimizar a prestação de serviço personalizado às demandas dos usuários. Além disso, o estudo possibilitou determinar o perfil de usuários atendidos durante uma década, o que permite compreender as características dos atendimentos fonoaudiológicos por todo esse período.

\section{CONCLUSÃO}

Em relação aos aspectos sociodemográficos, o perfil da amostra estudada revelou que a maioria das famílias era de baixa renda, constituída por bebês nascidos de prematuridade tardia, com complicações características desta condição. Os dados clínicos revelaram que a maioria das complicações observadas estiveram presentes nos prematuros extremos, com significância estatística para tipo de parto (cesáreo); peso, estatura e perímetro cefálico (os quais apresentaram menor mediana); uso de drogas ototóxicas; hemorragia intracraniana; sepse e icterícia. Quanto aos dados assistenciais, observou-se que a maior demanda da assistência foi para prematuros extremos, com significância estatística para dias na UTI e dias no Método Canguru.

\section{REFERÊNCIAS}

1. Beleza LDO, Ribeiro LM, Paula RAP, Guarda LEDA, Vieira GB, Costa KS. Perfil de recém-nascidos de risco atendidos por enfermeiros em seguimento ambulatorial: estudo de coorte retrospectiva. Rev Lat
Am Enfermagem. 2019;27:e3113. http://dx.doi.org/10.1590/15188345.2301 .3113 .

2. Worl Health Organization. Born too soon: the global action report on preterm birth. Geneva: WHO; 2012.

3. Ahishakiye A, Abimana MC, Beck K, Miller AC, Betancourt TS, Magge H, Mutaganzwa C, Kirk CM. Developmental outcomes of preterm and low birth weight toddlers and term peers in Rwanda. Ann Glob Health. 2019;85(1):147. http://dx.doi.org/10.5334/aogh.2629.

4. Nascimento GB, Kessler TM, Souza APR, Costa I, Moraes AB. Indicadores de risco para a deficiência auditiva e aquisição da linguagem e sua relação com variáveis socioeconômicas, demográficas e obstétricas em bebês pré-termo e a termo. CoDAS. 2020;32(1):e20180278. http:// dx.doi.org/10.1590/2317-1782/20192018278. PMid:32049152.

5. Stadio AD, Molini E, Gambacorta V, Giommetti G, Volpe AD, Ralli $\mathrm{M}$, et al. Sensorineural hearing loss in newborns hospitalized in neonatal intensive care unit: an observational study. Int Tinnitus J. 2019;23(1):31-6. http://dx.doi.org/10.5935/0946-5448.20190006. PMid:31469525.

6. ACRIAR. Projeto Acriar Ambulatório da Criança de Risco [Internet]. Belo Horizonte: ACRIAR; 2019 [acesso em 12 nov 2019]. Disponível em: https://www.medicina.ufmg.br/acriar/index.php

7. Montgomery KS. Apgar scores: examining the long-term significance. J PerinatEduc. 2000;9(3):5-9. http://dx.doi.org/10.1624/105812400X87716. PMid:17273212.

8. Pereira AYK, Ozela CMS, Ribeiro NM, Almeida ACV, Souza GTP. Seguimento ambulatorial de prematuros: acompanhamento nutricional. Pará Research Medical Journal. 2017;1(2):e17. http://dx.doi.org/10.4322/ prmj.2017.017.

9. Chermont AG, Silva EFA, Vieira CC, Souza LEC Fo, Matsumura ESS, Cunha KC. Fatores de risco associados à prematuridade e baixo peso ao nascer nos extremos da vida reprodutiva em uma maternidade privada. Rev Eletrônica Acervo Saúde. 2020;(39):e2110. http://dx.doi. org/10.25248/reas.e2110.2020.

10. Souza DML, Silva Maia LC, Zêgo ZDF, Jaeger GP, Maciel WS. Prevalência de prematuridade e fatores associados no estado do Rio Grande do Sul. Braz J Hea Rev. 2019;2(5):4052-70. http://dx.doi. org/10.34119/bjhrv2n5-014.

11. Schmidt B, Bolze SDA, Vieira ML, Crepaldi MA. Percepções Parentais sobre o Temperamento Infantil e suas Relações com as Variáveis Sociodemográficas das Famílias. Psicol Teor Pesqui. 2018;34(0):e3436. http://dx.doi.org/10.1590/0102.3772e3436.

12. Correa W, Minetto MF, Cappellaro-Kobren R, Kruszielski L. Crenças sobre práticas parentais em crianças em famílias de crianças com atraso no desenvolvimento. INFAD. 2018;3(1):21-30.

13. Amaral GR, Castanheira AM, Thomé MT, Silveira IMB. Tendência secular de crescimento em estatura de crianças e adolescentes e sua associação com fatores extrínsecos a partir da segunda metade do século XX no Brasil: uma revisão de literatura. Braz J of Develop. 2020;6(5):26971-81. http://dx.doi.org/10.34117/bjdv6n5-228.

14. Silveira RC, Procianoy RS. Preterm newborn's postnatal growth patterns: how to evaluate them. J Pediatr (Rio J). 2019;95(Suppl 1):42 8. http://dx.doi.org/10.1016/j.jped.2018.10.006. PMid:30521768.

15. Al-Qahtani B, Al-Otaibi M, Alabdulajabbar K, Selayem NB, Alshehri W, Omair A, et al. Retinopathy of prematurity incidence and risk factors in a Tertiary Hospital in Riyadh, Saudi Arabia. Middle East Afr J Ophthalmol. 2020;26(4):235. http://dx.doi.org/10.4103/meajo. MEAJO_131_18. PMid:32153336. 
16. Penha SC. Fatores de risco maternos associados à prematuridade em uma maternidade-escola. Sanare (Sobral, Online). 2019;18(2):43-51. http://dx.doi.org/10.36925/sanare.v18i2.1373.

17. Schmidt S, Norman M, Misselwitz B, Piedvache A, Huusom LD, Varendi $\mathrm{H}$, et al. Mode of delivery and mortality and morbidity for very preterm singleton infants in a breech position: a European cohort study. Eur J Obstet Gynecol Reprod Biol. 2019;234:96-102. http:// dx.doi.org/10.1016/j.ejogrb.2019.01.003. PMid:30682601.

18. Hadgu FB, Gebretsadik LG, Mihretu HG, Berhe AH. Prevalence and factors associated with neonatal mortality at ayder comprehensive specialized Hospital, Northern Ethiopia. A cross-sectional study. Pediatric Health, Med Ther. 2020;11:29-37.

19. Dortas ARF, Mello DMS, Bezerra LA, De Lima RG, Neves VHD, Aragão JA. Fatores de risco associados a sepse neonatal: artigo de revisão. Rev Eletrônica Acervo Cient. 2019;7:e1861. http://dx.doi. org/10.25248/reac.e1861.2019.

20. Queiroz MN, Gomes TGACB, Moreira ACG. Idade gestacional, índice de Apgar e peso ao nascer no desfecho de recém-nascidos prematuros. Com Ciênc Saúde (Porto Alegre). 2018;29(4):236-42.

21. Duarte-Sena MR, Ferrarini PB, Frare S, Cunha VSM, Silva RLF. Influência da posição canguru no sistema cardiopulmonar de prematuros em uma Unidade de Terapia Intensiva Neonatal na Amazônia. Rev Eletrônica Acervo Saúde. 2020;(41):e2419.

22. Basso CSD, Arroyo MAS, Saes MABF, Beani L, Maia AB, Lourenção LG. Breastfeeding rate and speech-language therapy in the Kangaroo Method. Rev CEFAC. 2019;21(5):e11719. http://dx.doi.org/10.1590/1982$0216 / 201921511719$.
23. Rodriguero CB, Ichisato SMT, Trombelli FSO, Macedo V, Oliveira MLF, Rossetto EG. Prematuro alimentado com leite humano versus leite humano acrescido de FM85®. Acta Paul Enferm. 2019;32(5):53845. http://dx.doi.org/10.1590/1982-0194201900075.

24. Nunes JA, Bianchini EMG, Cunha MC. Saturação de oxigênio e frequência cardíaca em prematuros: comparação entre as técnicas de copo e sonda-dedo. CoDAS. 2019;31(6):e20180221. http://dx.doi. org/10.1590/2317-1782/20192018221. PMid:31800880.

25. Carvalho BM, Tavares WR, Vicente JB, Sanguino GZ, Leite AM, Furtado MCC. Early access to biological neonatal screening: coordination among child care action programs. Rev Lat Am Enfermagem. 2020;28:e3266. 2020;28:e3266. http://dx.doi.org/10.1590/1518-8345.2938.3266.

26. Martinelli RLC, Marchesan IQ, Lauris JB, Honório HM, Gusmão RJ, Berretin-Felix G. Validade e confiabilidade da triagem: "teste da linguinha. Rev CEFAC. 2016;18(6):1323-31. http://dx.doi. org/10.1590/1982-021620161868716.

27. Marinho ACA, Pereira ECS, Torres KKC, Miranda AM, Ledesma ALL. Evaluation of newborn hearing screening program. Rev Saude Publica. 2020 Abr 30;54:44. PMid:32374803.

28. Brasil. Lei $\mathrm{n}^{0} 12.303$, de 2 de agosto de 2010. Lei do Teste da Orelhinha Dispõe sobre a obrigatoriedade de realização do exame denominado Emissões Otoacústicas Evocadas. Diário Oficial União; Brasília; 2 ago 2010; 1:1.

29. Brasil. Lei $\mathrm{n}^{\circ} 13.002$, de 20 de junho de 2014. Teste da linguinha. Obriga a realização do Protocolo de Avaliação do Frênulo da Língua em Bebês. Diário Oficial da União; Brasília; 20 jun 2014. 